Forecast Combinations for Value at Risk and Expected Shortfall

\author{
James W. Taylor \\ Saïd Business School \\ University of Oxford
}

2 April 2019

Address for Correspondence:

James W. Taylor

Saïd Business School

University of Oxford

Park End Street

Oxford OX1 1HP, UK

Tel: +44 (0)1865 288927

Email: james.taylor@sbs.ox.ac.uk 


\title{
Forecast Combinations for Value at Risk and Expected Shortfall
}

\begin{abstract}
Combining provides a pragmatic way to synthesise information provided by individual forecasting methods. In the context of forecasting the mean, numerous studies have shown that combining often leads to improved accuracy. Despite the importance of Value at Risk (VaR), few papers have considered quantile forecast combinations. A risk measure that is receiving increasing attention is Expected Shortfall (ES), which is the expectation of the exceedances beyond the VaR. There have been no previous studies on combining ES predictions, which is presumably due to there being no suitable loss function for the ES. However, it has recently been shown that a set of scoring functions exist for the joint estimation or backtesting of VaR and ES forecasts. We use such scoring functions to estimate combining weights for $\mathrm{VaR}$ and $\mathrm{ES}$ prediction. Results for five stock indices show that combining outperforms the individual methods for the $1 \%$ and $5 \%$ probability levels.
\end{abstract}

Keywords: Value at risk; expected shortfall; combining; elicitability; scoring functions. 


\section{Introduction}

As a measure of financial market risk, Value at Risk (VaR) has been widely used for regulatory purposes and internal risk management. VaR is a conditional quantile in the lower tail of the distribution of the return on a portfolio. Although straightforward to interpret, VaR has the limitation of providing no information regarding potential exceedances beyond the quantile. As an alternative risk measure, expected shortfall (ES) is receiving increasing attention, and is recommended as a risk measure by the Basel Committee on Banking Supervision (Basel Committee, 2016). ES is the conditional expectation of exceedances beyond the VaR. Artzner et al. (1999) point out that, in contrast to VaR, ES has the appealing property of subadditivity, which means that the measure for a portfolio cannot be greater than the sum of the measure for the constituent parts of the portfolio. An apparent disadvantage of ES is that it is not elicitable, which means that the correct ES forecast is not the unique minimiser of the expectation of any loss function. This presents a challenge to estimating and backtesting ES. To address this, Fissler and Ziegel (2016) have provided a set of joint loss functions for VaR and ES for which these two measures are jointly elicitable. In this paper, we use these loss functions in the context of forecast combinations.

The essential motivation for combining forecasts is that, when competing forecasts are available, a combination can enable a pragmatic synthesis of the information inherent in the individual predictions. Another perspective is that the combination provides a potentially diversified portfolio of the different forecasts. Since the seminal work of Bates and Granger (1969), a large literature has developed on combining forecasts of the conditional mean, with empirical support available across a variety of applications. An interesting empirical finding is that a simple average is typically very competitive. For forecasting the mean, least squares provides a natural approach to optimizing convex combining weights, or perhaps unconstrained weights in a model where individual forecasts can be viewed as regressors. Building on this, Granger (1989) and Granger et al. (1989) suggest that quantile forecasts 
could be combined using quantile regression. Taylor and Bunn (1998) consider the appeal of constraining the quantile regression parameters. They consider a zero intercept term and convex combining weights, which has been common for combinations of forecasts of the mean. Giacomini and Komunjer (2005) use the quantile regression framework to enable tests of quantile forecast encompassing, which provides theoretical justification for combining in cases where one forecast does not encompass another. Shan and Yang (2009) calculate weights based on the inverse of the quantile regression loss function.

In the VaR context, given the variety of different quantile forecasting methods available, it is perhaps surprising that there has not been more consideration of forecast combinations. In their recent review of the VaR literature, Nieto and Ruiz (2016) report just a handful of studies on combining. McAleer et al. (2013a, 2013b) look at selecting the maximum, minimum or median of a set of forecasts. Halbleib and Pohlemeier (2012) derive combining weights by maximizing conditional coverage, as well as quantile regression. Jeon and Taylor (2013) and Fuertes and Olmo (2013) also use quantile regression. They combine individual forecasts constructed from different information sources, including historical daily returns, option-implied volatility, realized volatility and the intraday range.

Although in recent years there has been an increased interest in forecasting ES, we are not aware of studies that have looked at combining ES forecasts. This is presumably due to ES not being elicitable. In this paper, we propose the use of Fissler and Ziegel's (2016) joint $\mathrm{VaR}$ and ES loss functions to estimate combining weights for $\mathrm{VaR}$ and ES prediction. Elliott and Timmermann (2004) show that the issue of whether combining weights should be equal depends on the loss function, implying that empirical evidence from the literature on forecasting the mean, where a squared loss function is appropriate, may not transfer to VaR and ES forecasting. Our paper provides some empirical results regarding this.

If $\mathrm{VaR}$ and $\mathrm{ES}$ predictions are obtained from density forecasts, an alternative to combining VaR and ES predictions is to combine the density forecasts (see, for example, Hall 
and Mitchell, 2007; Jore et al., 2010). Opschoor et al. (2017) describe how the combining method can be adapted to focus on a particular part of the density, such as the left tail when $\mathrm{VaR}$ and $\mathrm{ES}$ are of interest. However, this combining approach is not of use when combining one or more $\mathrm{VaR}$ and ES forecasting methods that are not based on density forecasts. This is our interest in this paper. For example, VaR and ES forecasts could be produced by autoregressive quantile or expectile models, and indeed this is the case in our empirical analysis. We feel that it is important to consider this more general case, because forecast combinations are particularly useful when forecasts are produced by methods that are based on different information or use the information in notably different ways.

Section 2 briefly reviews loss functions for VaR and ES prediction. Section 3 describes two combining formulations that we propose. Section 4 presents an empirical study based on daily stock indices. Section 5 provides a simulation study. Section 6 summarises and concludes the paper.

\section{Scoring Functions for VaR and ES}

A scoring function is the term used in decision theory for a loss function when used to evaluate a forecast of some measure of a probability distribution, such as a quantile. As we explained in the previous section, the measure is described as elicitable if the correct forecast of the measure is the unique minimiser of the expectation of at least one scoring function. Such scoring functions are called strictly consistent for the measure (Fissler and Ziegel, 2016). A strictly consistent scoring function can be used as the loss function in model estimation (Gneiting and Raftery, 2007). We now describe strictly consistent scoring functions that we propose to use to estimate forecast combining weights for VaR and ES. 


\subsection{Scoring Functions for VaR}

$\mathrm{VaR}$ is an elicitable risk measure. Strictly consistent scoring functions for VaR are of the following form (Gneiting and Raftery, 2007):

$$
S\left(Q_{t}, y_{t}\right)=\left(\alpha-I\left(y_{t} \leq Q_{t}\right)\right)\left(G\left(y_{t}\right)-G\left(Q_{t}\right)\right),
$$

where $y_{t}$ is the variable of interest; $Q_{t}$ is the quantile with probability level $\alpha$; I is the indicator function; and $G$ is a weakly increasing function. Selecting $G$ to be the identity function leads to the quantile score of the following expression:

$$
S\left(Q_{t}, y_{t}\right)=\left(\alpha-I\left(y_{t} \leq Q_{t}\right)\right)\left(y_{t}-Q_{t}\right) .
$$

This score is widely used in the VaR literature due to its simplicity, and its familiarity as the quantile regression loss function. Averaging the score across a sample gives a measure for evaluating quantile forecasts.

\subsection{Joint Scoring Functions for VaR and ES}

The ES is not an elicitable risk measure (Gneiting, 2011), meaning that a suitable scoring function does not exist for the sole purpose of estimating or evaluating ES forecasts. However, a measure that is not elicitable individually can be jointly elicitable with another measure. This is the case for the variance, which is only jointly elicitable (with the mean). With regard to the ES, Fissler and Ziegel (2016) prove that it is jointly elicitable with the VaR. They show that strictly consistent scoring functions, for jointly evaluating VaR and ES forecasts, are of the following form:

$$
\begin{aligned}
S\left(Q_{t}, E S_{t}, y_{t}\right)=\left(I\left(y_{t} \leq Q_{t}\right)-\alpha\right) G_{1}\left(Q_{t}\right)-I\left(y_{t} \leq Q_{t}\right) G_{1}\left(y_{t}\right) \\
\quad+G_{2}\left(E S_{t}\right)\left(E S_{t}-Q_{t}+I\left(y_{t} \leq Q_{t}\right)\left(Q_{t}-y_{t}\right) / \alpha\right)-\zeta_{2}\left(E S_{t}\right)+a\left(y_{t}\right),
\end{aligned}
$$

where $E S_{t}$ is the ES; and $G_{1}, G_{2}, \zeta_{2}$ and $a$ are functions satisfying a number of conditions, including the properties that $G_{2}=\zeta_{2}^{\prime}, G_{1}$ is increasing, and $\zeta_{2}$ is increasing and convex. (The domain of $\zeta_{2}$ contains only negative values, because we are considering $\alpha<50 \%$, which 
implies that $E S_{t}$ is negative.) Fissler and Ziegel (2016) note that the scoring function in expression (2) remains strictly consistent for the case of $G_{1}=0$ and $\zeta_{2}$ strictly increasing and convex. In expression (2), the terms involving $G_{1}$ are collectively a strictly consistent scoring function for a quantile, with the other terms assessing both the quantile and ES (Fissler et al., 2016). Therefore, to reduce the emphasis on quantile accuracy, one can set $G_{1}=0$, and indeed this has been the choice in several studies. Table 1 presents four scoring functions, of the form of expression (2), that have been proposed. We discuss them in the rest of this section.

\section{Table 1}

Functions used within the joint $\mathrm{VaR}$ and ES scoring function of expression (2) to give four different versions of the score: AL, NZ, FZG and AS scores.

\begin{tabular}{ccccc}
\hline & $G_{1}(x)$ & $G_{2}(x)$ & $\zeta_{2}(x)$ & $a(y)$ \\
\hline $\mathrm{AL}$ & 0 & $-1 / x$ & $-\ln (-x)$ & $1-\ln (1-\alpha)$ \\
$\mathrm{NZ}$ & 0 & $1 / 2(-x)^{-1 / 2}$ & $-(-x)^{1 / 2}$ & 0 \\
$\mathrm{FZG}$ & $x$ & $\exp (x) /(1+\exp (x))$ & $\ln (1+\exp (x))$ & $\ln (2)$ \\
$\mathrm{AS}$ & $-1 / 2 W x^{2}$ & $\alpha x$ & $1 / 2 \alpha x^{2}$ & 0 \\
\hline
\end{tabular}

Taylor (2019) points out that, if $G_{1}=0, G_{2}=-1 / x, \zeta_{2}(x)=-\ln (-x)$ and $a=1-\ln (1-\alpha)$, the scoring function is equal to the negative of the log-likelihood function of an asymmetric Laplace (AL) density with time-varying location and scale parameters. Using this scoring function for model estimation has some appeal because it can be viewed as a relatively minor extension of quantile regression, which is equivalent to maximizing an AL likelihood with time-varying location and constant scale. We refer to Taylor's (2019) proposed score as the AL score. Taylor (2019) uses the score to estimate dynamic joint models for VaR and ES, and this proposal is given theoretical support by the recent work of Patton et al. (2019).

Nolde and Ziegel (2017) consider comparative backtests for risk measures. Their numerical study essentially uses the AL score, and also the score resulting from setting $G_{1}=0$, $G_{2}=1 / 2(-x)^{-1 / 2}, \zeta_{2}(x)=-(-x)^{1 / 2}$ and $a=0$ in expression (2), which we refer to as the NZ score. 
In their empirical analysis, Fissler et al. (2016) use the scoring function produced by using the following functions in expression (2): $G_{1}(x)=x, \quad G_{2}=\exp (\mathrm{x}) /(1+\exp (\mathrm{x}))$, $\zeta_{2}(x)=\ln (1+\exp (x))$ and $a=0$. In our empirical work, we found that the first three significant figures of the values of this score did not differ between forecasting methods. This meant that it was difficult to distinguish between the methods when comparing relative measures, which we computed in order to average the performance across a set of stock indices, as we describe in detail in Section 4.3.2. To make the relative measures more easily comparable, we set $a=\ln (2)$ in expression (2). We refer to this as the $F Z G$ score.

Another example of a joint scoring function is proposed by Acerbi and Székeley (2014). We refer to it as the $A S$ score. It is produced by setting $G_{1}(x)=-1 / 2 W x^{2}, G_{2}(x)=\alpha x$, $\zeta_{2}(x)=1 / 2 \alpha x^{2}$ and $a=0$ in expression (2). Fissler and Ziegel (2016) explain that the score is strictly consistent, provided the parameter $W$ is chosen such that $W Q_{t}<E S_{t}$. (Recall that $E S_{t}<0$ and $Q_{t}<0$ because $\alpha<50 \%$.) In our empirical analysis, we used $W=4$, as this was the smallest integer that ensured $W Q_{t}<E S_{t}$ for all pairs of forecasts of $E S_{t}$ and $Q_{t}$ from all methods in our study. We did not use the AS score for estimation, as we could not guarantee that our chosen value of $W$ would lead to $W Q_{t}<E S_{t}$ for all resulting pairs of forecasts of $E S_{t}$ and $Q_{t}$.

We present the AL, NZ, FZG and AS scoring functions in Table 1. Our proposal is to use the first three of these to estimate forecast combining weights for the prediction of $\mathrm{VaR}$ and ES. In using such joint scoring functions for estimation, our work has similarities to that of Taylor (2019) and Patton et al. (2019), who use the AL score to estimate dynamic models, and Dimitriadis and Bayer (2017), who present a regression framework for VaR and ES.

\section{Methods for Combining Forecasts}

\subsection{Minimum Score Combining}

In this paper, we address the situation where we have a set of individual methods, each producing a forecast for the VaR and ES. As the quality of a method's VaR and ES 
forecasts may differ, it seems desirable to allow the combining weights to differ for the VaR and ES. However, as the ES is equal to the sum of the VaR and the mean of the exceedances beyond the $\mathrm{VaR}$, it is not possible to distinguish VaR accuracy from ES accuracy. In view of this, our proposal is a formulation that does not combine ES forecasts, but instead combines forecasts of the difference between ES and VaR. We give the name spacing to this difference. We refer to the method as minimum score combining, and express it as follows:

$$
\begin{aligned}
& \hat{Q}_{c t}=\sum_{i=1}^{M} w_{i}^{Q} \hat{Q}_{i t}, \\
& \hat{E S_{c t}}=\hat{Q}_{c t}+\sum_{i=1}^{M} w_{i}^{S}\left(\hat{E S} \hat{S}_{i t}-\hat{Q}_{i t}\right),
\end{aligned}
$$

where $M$ is the number of individual methods; $\hat{Q}_{i t}$ is the quantile forecast and $\hat{E S}$ it is the ES forecast produced by the $i$ th individual method; $\hat{Q}_{c t}$ is the combined quantile forecast; $\hat{E S}{ }_{c t}$ is the combined ES forecast; $w_{i}^{Q}$ is the combining weight for the quantile forecast from the $i$ th method; and $w_{i}^{S}$ is the combining weight for the spacing between ES and quantile forecasts from the $i$ th method. We constrain the $w_{i}^{Q}$ to be non-negative and sum to 1 , and we impose the same constraints on the $w_{i}^{S}$. In addition to convex weights being common and intuitively appealing, they ensure that $\hat{E S}_{c t}$ will exceed $\hat{Q}_{c t}$. This is not easily ensured if $\hat{E S}_{c t}$ is constructed as a convex combination of the individual ES forecasts.

We estimated both sets of combining weights, $w_{i}^{Q}$ and $w_{i}^{S}$, in a single step by minimising a chosen scoring function. The optimal combining weights are those that lead to in-sample estimates for $\hat{Q}_{c t}$ and $\hat{E S}_{c t}$ that minimise the scoring function. We describe the minimisation further in Section 4.2. 


\subsection{Relative Score Combining}

A simple method, used to combine forecasts of the mean, is to set convex combining weights to be inversely proportional to the mean squared error (MSE) (see Bates and Granger, 1969). This has the appeal of robustness when the estimation sample is small or there are many predictors (see, for example, Stock and Watson, 2001). Shan and Yang (2009) use the approach to combine quantile forecasts. To measure accuracy, instead of MSE, they use the quantile score. We apply this idea to our VaR and ES context, by using the joint scoring functions of expression (2) to measure accuracy. The method leads to a single set of weights $w_{i}$ for both $\mathrm{VaR}$ and $\mathrm{ES}$ prediction. We refer to the method as relative score combining, and present it as follows:

$$
\begin{aligned}
& \hat{Q}_{c t}=\sum_{i=1}^{M} w_{i} \hat{Q}_{i t}, \\
& \hat{E S}_{c t}=\sum_{i=1}^{M} w_{i} \hat{E S_{i t}} \\
& w_{i}=\frac{\exp \left(-\lambda \sum_{j=1}^{t-1} S\left(\hat{Q}_{i j}, \hat{E S}_{i j}, y_{j}\right)\right)}{\sum_{k=1}^{M} \exp \left(-\lambda \sum_{j=1}^{t-1} S\left(\hat{Q}_{k j}, \hat{E S_{k j}, y_{j}}\right)\right)},
\end{aligned}
$$

where $S$ is the chosen joint scoring function, which, for each method $i$, is computed in each period $j$ and then summed for all $t$ - 1 in-sample observations; and $\lambda>0$ is a tuning parameter, included in the combining formulations of Shan and Yang (2009) and Stock and Watson (2001) to control how much the combining weights depend on the scoring function. A value of $\lambda$ close to zero reduces the method to the simple average, while a high value of $\lambda$ results in the selection of the individual method with the best historical accuracy. In our work, we optimised the value of $\lambda$ by minimising the in-sample values of a chosen scoring function. We describe the optimisation further in Section 4.2. 


\section{Empirical Analysis}

Our empirical study considered the day-ahead forecasting of the $1 \%$ and $5 \% \mathrm{VaR}$ and ES for daily log-returns of the following five stock indices: CAC 40, DAX 30, FTSE 100, NIKKEI 225 and S\&P 500. We downloaded the data from Bloomberg. Each series consisted of the 6000 daily observations ending on 31 May 2017. Due to different holiday periods in each country, the start dates differed for the five indices. The start dates were 26 October 1993, 27 September 1993, 1 September 1993, 4 January 1993, and 4 August 1993 for the CAC 40, DAX 30, FTSE 100, NIKKEI 225 and S\&P 500, respectively. Fig. 1 shows the FTSE 100 returns, with the financial crisis evident around $2008 .^{1}$

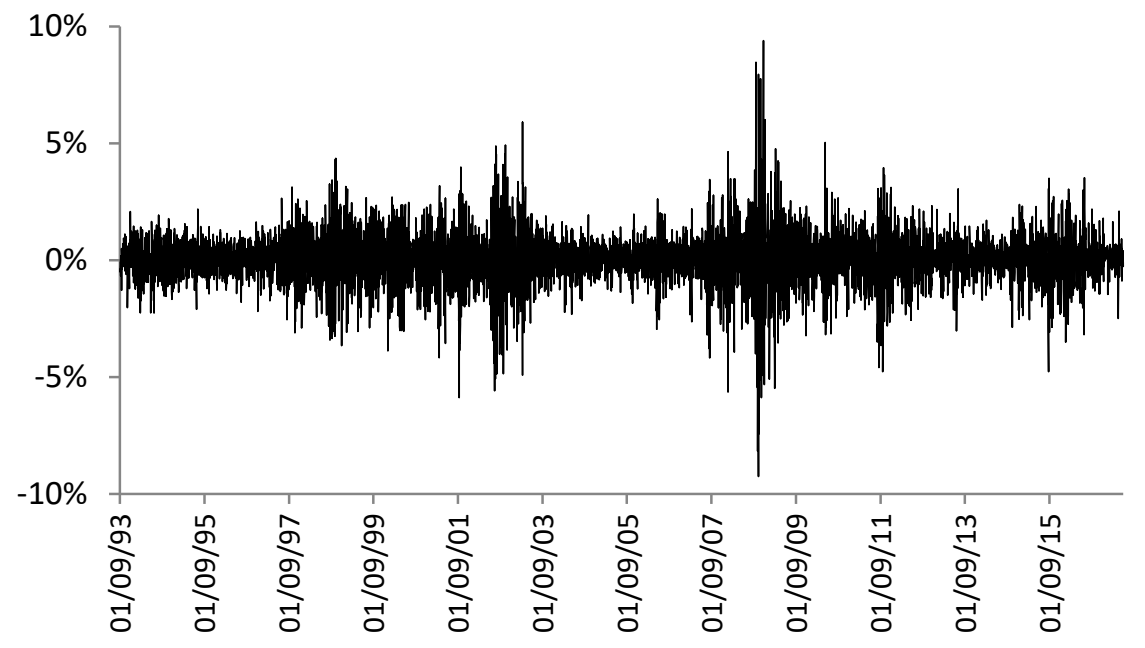

Fig. 1. The series of 6000 FTSE 100 returns ending on 31 May 2017.

We used a rolling window of 2000 days, which we moved forward by one day at a time, for repeated re-estimation of the parameters of the individual forecasting methods. This enabled us to produce out-of-sample forecasts from each of these methods for the final 4000 days in each series. Our combining methods focused on this period of 4000 days, with a rolling window of 2000 days used for repeated re-estimation of the combining weights. The final 2000 days was used to compare the out-of-sample forecast accuracies of all the methods. Prior to the application of the VaR and ES estimation methods, we applied an

\footnotetext{
${ }^{1}$ Our decision to plot the FTSE 100 was made arbitrarily. The time series of the other four indices showed similar features.
} 
autoregressive model of order 1 as an initial filter. The parameters of this filter were estimated using each rolling window of 2000 returns.

\subsection{Individual Methods}

As combining has the greatest potential when the individual methods have used different information or have used information in differing ways, we implemented a diverse set of individual methods, including nonparametric, parametric and semiparametric time series methods, as well as a method based on intraday data. We now describe these methods.

\subsubsection{Historical Simulation}

As a simple nonparametric method, we used historical simulation based on the 250 observations up to and including the forecast origin. We also considered the use of 100, 500 and 2000 observations, but this did not lead to an overall improvement in forecast accuracy.

\subsubsection{GJR-GARCH}

As a common parametric method, we implemented a GJR-GARCH(1,1) model based on a Student $t$ distribution. This asymmetric model was notably more accurate than a GARCH $(1,1)$ model. We also considered filtered historical simulation, which applied historical simulation to the standardised residuals, as well as the method of McNeil and Frey (2000), which applies peaks-over-threshold extreme value theory (EVT) to the standardised residuals. However, these methods did not deliver a substantial improvement, and so we used the Student $t$ distribution, as this allowed us to have a fully parametric approach in our study.

\subsubsection{CAViaR-AS-EVT}

Conditional autoregressive value at risk (CAViaR) models are autoregressive quantile models estimated using quantile regression (see Engle and Manganelli, 2004). Although 
directly modelling $\mathrm{VaR}$ is appealing, it provides no insight regarding the ES. This is addressed by Manganelli and Engle (2004) who estimate a CAViaR model for the $7.5 \%$ quantile, and then apply peaks-over-threshold EVT to the exceedances after standardising by the corresponding quantile estimates. The fitted extreme value distribution is then used to obtain the VaR and ES estimates. We implemented this approach, and in view of the superior performance of the asymmetric GJR-GARCH model relative to the GARCH model, we used the asymmetric slope (AS) CAViaR model, which we present in the following expression ${ }^{2}$ :

$$
Q_{t}=\beta_{0}+\beta_{1} I\left(y_{t-1}>0\right)\left|y_{t-1}\right|+\beta_{2} I\left(y_{t-1} \leq 0\right)\left|y_{t-1}\right|+\beta_{3} Q_{t-1} .
$$

\subsubsection{CARE-AS}

Expectiles are estimated by asymmetric least squares. They generalise the mean, just as quantiles generalise the median (Nolde and Ziegel, 2017). They were introduced by Newey and Powell (1987), who explain that "expectiles are determined by tail expectations in the same way that quantiles are determined by the distribution". The computational convenience of expectiles motivated Efron (1991) to suggest that they could be used to approximate quantiles. Drawing on this, Taylor (2008) proposes the use of a conditional autoregressive expectile (CARE) model to estimate VaR, and, as the ES can be expressed as a simple function of the expectile, the CARE model can also be used to deliver a forecast for the ES. We implemented this approach in our study. An important issue is the choice of $\tau$ expectile to use to approximate the $\alpha$ quantile. To optimise $\tau$, we followed the approach of Taylor (2008), which involves repeatedly re-estimating CARE models, reducing the $\tau$ by 0.0001 each time, until the proportion of in-sample exceedances beyond the fitted expectile is

\footnotetext{
${ }^{2}$ Estimation of the parameters $\beta_{i}$ proceeded by first sampling $10^{4}$ candidate parameter vectors from uniform distributions with lower and upper bounds based on initial experimentation. We also included, as an additional candidate, the parameter vector that had been optimised for the previous window of observations. From the set of candidate vectors, the three giving the lowest value of the quantile score were used, in turn, as the initial vector in a quasi-Newton algorithm. The resulting vector, with lowest score, was chosen as the final parameter vector.
} 
closer to $\alpha$ than a predefined tolerance. We started this procedure with values of $\tau=0.0018$ and $\tau=0.0167$ for the $1 \%$ and $5 \%$ probability levels, respectively. These values were chosen after initial experimentation. In view of our choice of asymmetric GARCH and CAViaR models, we used the following asymmetric slope (AS) CARE model:

$$
\mu_{t}=\beta_{0}+\beta_{1} I\left(y_{t-1}>0\right)\left|y_{t-1}\right|+\beta_{2} I\left(y_{t-1} \leq 0\right)\left|y_{t-1}\right|+\beta_{3} \mu_{t-1},
$$

where $\mu_{t}$ is the expectile. We estimated the parameters $\beta_{i}$ using the same approach that we described in Section 4.1.3 for the CAViaR model, with the one difference being that we replaced the quantile score with the following expectile score:

$$
S\left(\mu_{t}, y_{t}\right)=\left|\tau-I\left(y_{t} \leq \mu_{t}\right)\right|\left(y_{t}-\mu_{t}\right)^{2} .
$$

\subsubsection{HAR-Range}

Intraday data has been found to be useful in estimating features of the distribution of daily returns. For example, realized volatility has been widely used as a basis for forecasting daily volatility. The heterogeneous autoregressive (HAR) model of realized volatility is a simple and pragmatic approach, where a volatility forecast is constructed from the realized volatility over different time horizons (Corsi, 2009). However, intraday data can be expensive, and resources are required for pre-processing. Given the ready availability of the daily high and low price, an alternative way to capture intraday volatility is to use the intraday range (see, for example, Alizadeh et al., 2002; Gerlach and Chen, 2014). We take this approach, and follow Brownlees and Gallo (2010) by implementing the HAR model with realized volatility replaced by the intraday range, as in the following expressions:

$$
\begin{aligned}
& \text { Range }_{t}=\beta_{1}+\beta_{2} \text { Range }_{t-1}+\beta_{3} \text { Range }_{t-1}^{w}+\beta_{4} \text { Range }_{t-1}^{m}+\varepsilon_{t}, \\
& \text { Range }_{t-1}^{w}=\frac{1}{5} \sum_{i=1}^{5} \text { Range }_{t-i}, \\
& \text { Range }_{t-1}^{m}=\frac{1}{22} \sum_{i=1}^{22} \text { Range }_{t-i},
\end{aligned}
$$


where Range $_{t}$ is the difference between the highest and lowest log price on day $t$; Range ${ }_{t-1}^{w}$ and Range $_{t-1}^{m}$ are averages of Range $e_{t}$ over a week and month, respectively; $\varepsilon_{t}$ is an i.i.d. error term with zero mean; and the $\beta_{i}$ are parameters estimated using least squares. The conditional variance is then expressed as a linear function of the square of Range, with intercept and coefficient estimated using maximum likelihood based on a Student $t$ distribution. This model is then used to produce a variance forecast. To obtain VaR and ES forecasts, the forecast of the standard deviation is multiplied by the VaR and ES of the Student $t$ distribution.

\subsection{Combining Methods}

We combined forecasts using the minimum score and relative score methods of Section 3, as well as simple averaging. We combined two different sets of forecasts. The first included all five individual methods in Section 4.1. Even though the historical simulation method is known to be uncompetitive (see, for example, Chen et al., 2012), we included it in the combination in order to check that the relative score and minimum score combining methods would produce sets of weights that would account for the weakness of historical simulation. The outcome should be that these combining methods outperform the simple average. Nevertheless, including a poor method in a combination unnecessarily increases parameter estimation error, which can lead to a detrimental impact on accuracy. Indeed, including a poor method is unlikely to be done in practice. In view of this, we also applied the combining methods to a second set of individual methods that included just the four sophisticated methods, with the exclusion of historical simulation.

To estimate the combining weights, we used four different approaches, corresponding to the minimisation of the quantile score and the AL, NZ and FZG joint scoring functions. We obtained similar results when optimising with each of the joint scoring functions, and these results were better, or no worse, than those obtained by optimising the quantile score. In 
view of this, to save space, we report only the results produced using the AL score. ${ }^{34}$

In the minimum score combining method of expressions (3) and (4), we experimented with setting $w_{i}^{S}=w_{i}^{Q}$ for each $i$, which implies the use of the same set of combining weights for the VaR and ES. The results were quite similar to those without the constraint, and so, to save space, we report the results for only the unconstrained minimisation.

Figs. 2 and 3 present minimum score combining weights for a combination of the five individual methods for the 5\% probability level of the FTSE 100. Figs. 2 and 3 plot the VaR and ES combining weights, respectively. ${ }^{5}$ For each of the 2000 out-of-sample periods, the figures show the weights estimated by minimising the AL score using the 2000 observations up to and including the forecast origin. Fig. 2 also shows the minimised in-sample AL score plotted against the secondary $y$-axis. Although the ES weights are reasonably volatile over the out-of-sample period, the minimised AL score evolves smoothly, providing reassurance that the volatile ES weights are not due to a faulty optimisation procedure. For most of the out-ofsample period, CAViaR-AS-EVT and HAR-Range have the largest combining weights in Figs. 2 and 3.

The corresponding combining weights for the relative score method are shown in Fig. 4. Recall that, for this method, the set of weights for VaR combining is the same as the set for ES combining. The figure shows GJR-GARCH and HAR-Range with the largest weights for the first half of the plot. Interestingly, historical simulation has non-zero weights in Figs. 2 to 4, even though, as we show in Section 4.3, it is the least accurate of the individual methods.

\footnotetext{
${ }^{3}$ For the combining methods, we used a similar optimisation approach to the one described in Section 4.1.3 for the CAViaR model. For minimum score combining, we used $10^{5}$ candidate parameter vectors, with entries sampled from uniform distributions between 0 and 1 . We also included, as a candidate, the parameter vector that had been optimised for the previous window of observations. The 10 candidate vectors giving the lowest value of the scoring function were then each used as the initial vector in a quasi-Newton algorithm. The resulting vector, with lowest scoring function, was chosen as the final parameter vector. For relative score combining, which has just the one parameter $\lambda$, we used $10^{4}$ candidate values.

${ }^{4}$ An online appendix contains out-of-sample results for the combining methods with weights estimated using the quantile score, and the NZ and FZG scoring functions.

${ }^{5}$ Our decision to focus here on the FTSE 100 was made arbitrarily. We do not present the corresponding figures for the other four indices, as this would use considerable space, and would not provide significant useful additional insight.
} 


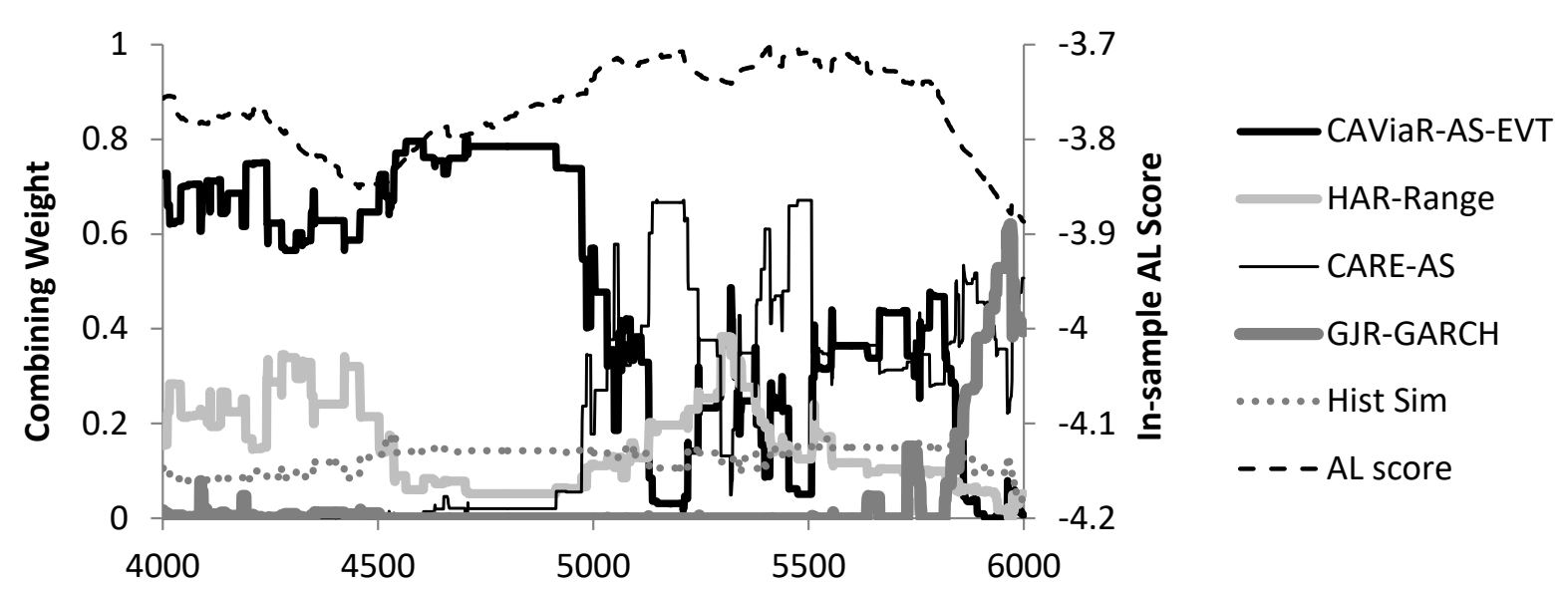

Fig. 2. For the 5\% probability level of the FTSE 100, minimum score combining weights $w_{i}^{Q}$ for the VaR combining of expression (3) used to combine five methods. Weights optimised by minimising the in-sample AL score, which is also shown in the plot.

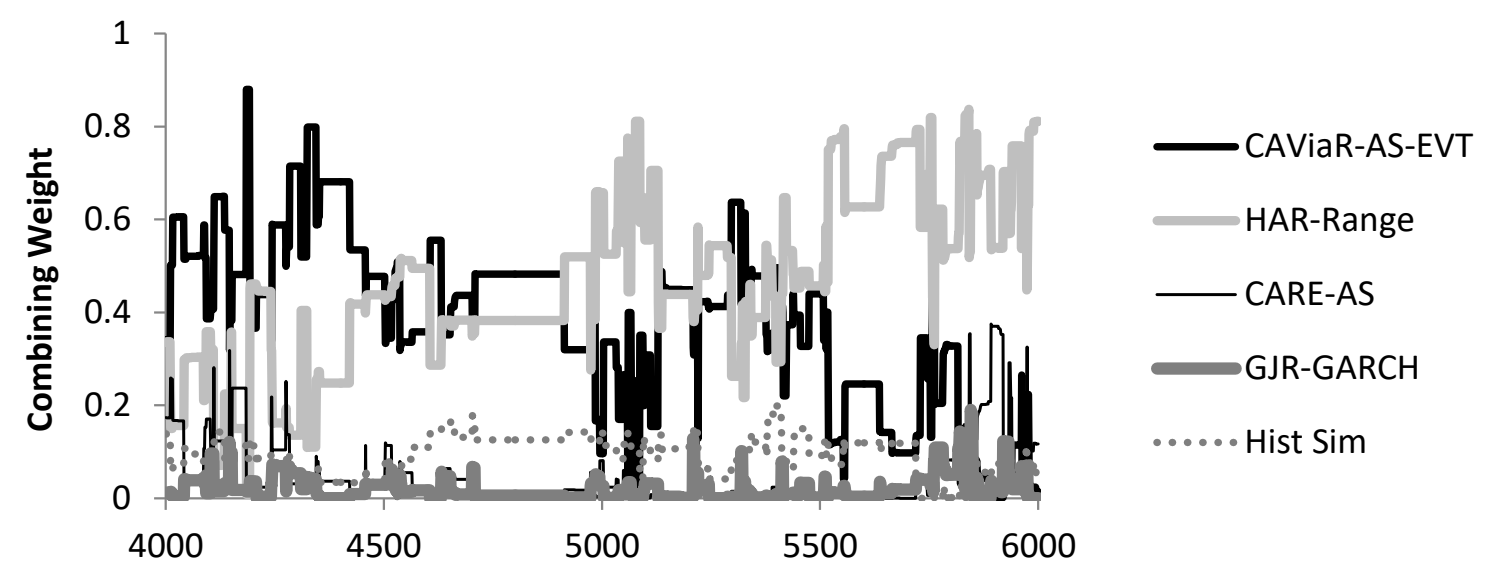

Fig. 3. For the 5\% probability level of the FTSE 100, minimum score combining weights $w_{i}^{S}$ for the spacings combination of expression (4) used to combine five methods. Weights optimised by minimising the in-sample AL score.

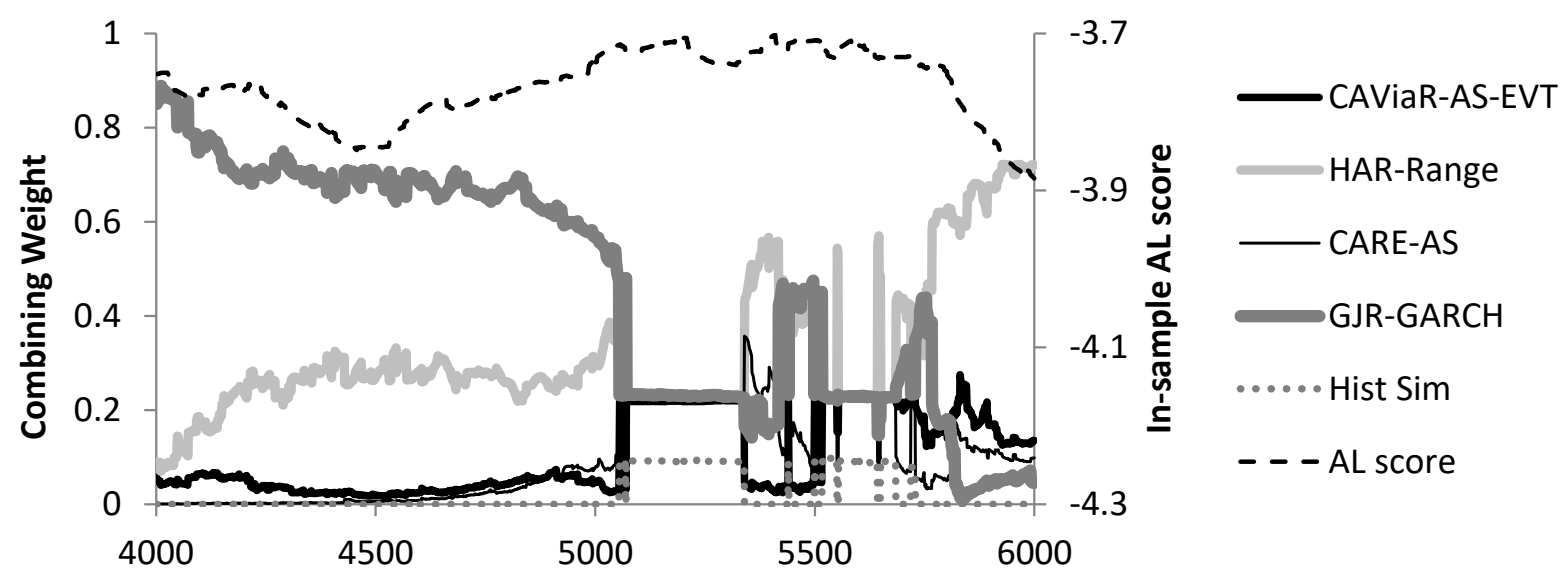

Fig. 4. For the 5\% probability level of the FTSE 100, relative score combining weights of expressions (5) to (7) used to combine five methods. Method optimised by minimising the insample AL score, which is also shown in the plot. 


\subsection{Backtesting VaR and ES Forecasts}

Traditionally, VaR and ES forecasts have tended to be evaluated using tests that Nolde and Ziegel (2017) describe as unconditional and conditional calibration tests. We use these in Section 4.3.1, and then consider scoring functions in Sections 4.3.2. Our out-ofsample evaluation focuses on the final 2000 periods of each series, as we have out-of-sample forecasts for each of these periods from all the individual and combining methods.

\subsubsection{Backtesting VaR and ES Forecasts with Calibration Tests}

$\mathrm{VaR}$ forecasts are typically evaluated using calibration tests. For probability level $\alpha$, a

quantile forecast $\hat{Q}_{t}$, is unconditionally calibrated if the variable $H_{t} t_{t}=\alpha-I\left(y_{t} \leq \hat{Q}_{t}\right)$ has zero unconditional expectation, and is conditionally calibrated if Hit has zero conditional expectation. We evaluated unconditional calibration using a test based on the binomial distribution to test whether Hit $t_{t}$ was significantly different from zero. Following common practice, our calibration testing did not attempt to incorporate parameter estimation error (see Escanciano and Olmo, 2010). In Table 2, for the $1 \%$ and 5\% probability levels, we summarise the test results for the five indices in the columns labelled VaR hit $\%$. The values reported are the number of indices for which the test was significant at the 5\% significance level. Throughout Table 2, lower values are better. There are non-zero entries for only the historical simulation, GJR-GARCH and HAR-Range methods.

To test for VaR conditional calibration, we implemented Engle and Manganelli's (2004) dynamic quantile test with four lags in the test's regression. We summarise the results in Table 2 for the five indices. For the $1 \%$ probability level, the historical simulation and HAR-Range methods are the worst performing methods, while for the $5 \% \mathrm{VaR}$, the poorest results are for historical simulation. 
Table 2

Results of calibration tests for the five stock indices.

\begin{tabular}{|c|c|c|c|c|c|c|}
\hline & \multicolumn{3}{|c|}{$1 \%$ probability level } & \multicolumn{3}{|c|}{$5 \%$ probability level } \\
\hline & $\begin{array}{l}\text { VaR } \\
\text { hit \% }\end{array}$ & $\begin{array}{c}\text { VaR } \\
\text { dynamic } \\
\text { quantile }\end{array}$ & $\begin{array}{c}\text { ES } \\
\text { bootstrap } \\
\text { test }\end{array}$ & $\begin{array}{l}\text { VaR } \\
\text { hit \% }\end{array}$ & $\begin{array}{c}\text { VaR } \\
\text { dynamic } \\
\text { quantile }\end{array}$ & $\begin{array}{c}\text { ES } \\
\text { bootstrap } \\
\text { test }\end{array}$ \\
\hline \multicolumn{7}{|l|}{ Individual methods } \\
\hline Historical Simulation & 3 & 5 & 3 & 0 & 5 & 1 \\
\hline GJR-GARCH & 1 & 0 & 1 & 0 & 0 & 3 \\
\hline HAR-Range & 2 & 2 & 0 & 1 & 0 & 1 \\
\hline CARE-AS & 0 & 0 & 0 & 0 & 1 & 0 \\
\hline CAViaR-AS-EVT & 0 & 0 & 0 & 0 & 0 & 0 \\
\hline \multicolumn{7}{|l|}{ Combining all } \\
\hline Simple average & 0 & 1 & 0 & 0 & 0 & 0 \\
\hline Relative score & 0 & 0 & 0 & 0 & 0 & 1 \\
\hline Minimum score & 0 & 0 & 0 & 0 & 0 & 1 \\
\hline \multicolumn{7}{|c|}{ Combining all except Historical Simulation } \\
\hline Simple average & 0 & 0 & 1 & 0 & 0 & 0 \\
\hline Relative score & 0 & 0 & 0 & 0 & 0 & 1 \\
\hline Minimum score & 0 & 0 & 0 & 0 & 0 & 1 \\
\hline
\end{tabular}

Notes: The values presented are the number of indices for which the test was significant at the $5 \%$ significance level. Low values are better, indicating a smaller number of tests for which calibration was rejected. The tests are described in Section 4.3.1.

To evaluate the ES forecasts, we followed the approach of McNeil and Frey (2000) by testing for zero mean in the discrepancy between the observed return and the ES forecast for the periods in which the return exceeds the $\mathrm{VaR}$ forecast. We standardised by dividing each discrepancy by the corresponding VaR estimate. The test examines whether the standardised discrepancies have zero unconditional expectation. To avoid any distributional assumptions about the distribution of the standardized discrepancies, we used the dependent circular block bootstrap used by Jalal and Rockinger (2008). The results are presented in Table 2. The table shows historical simulation performing relatively poorly for the $1 \%$ probability level, while the GJR-GARCH model was the worst performing for the 5\% probability level. 


\subsubsection{Backtesting VaR and ES Forecasts with Scoring Functions}

To evaluate VaR forecast accuracy, we calculated the quantile score. We then calculated the quantile skill score for each method as the ratio of the score to that of the historical simulation method, then subtracted this ratio from 1, and multiplied the result by 100. We report the skill scores in Table 3 for the $1 \%$ probability level. For all skill scores, higher values are preferable. To summarise performance across the five stock indices, we calculated the geometric mean of the ratios of the score for each method to the score for the historical simulation reference method, then subtracted this from one, and multiplied the result by 100 . The resulting values are presented in the final column of the table.

\section{Table 3}

$1 \% \mathrm{VaR}$ evaluated using quantile skill score $(\%)$.

\begin{tabular}{lcccccc}
\hline & CAC & DAX & FTSE & NIKKEI & S\&P & $\begin{array}{c}\text { Geo. } \\
\text { mean }\end{array}$ \\
\hline Individual methods & & & & & & \\
$\quad$ Historical Simulation & 0.0 & 0.0 & 0.0 & 0.0 & 0.0 & 0.0 \\
GJR-GARCH & 16.4 & 16.5 & 21.1 & 22.0 & 24.4 & 20.0 \\
HAR-Range & 18.3 & 20.6 & 20.2 & 18.8 & 26.4 & 20.8 \\
CARE-AS & 17.8 & 18.7 & 21.9 & 21.0 & 25.4 & 20.9 \\
$\quad$ CAViaR-AS-EVT & 17.0 & 17.3 & 21.3 & 21.5 & 23.7 & 20.1 \\
\hline Combining all & & & & & & \\
$\quad$ Simple average & 18.9 & 20.6 & 21.5 & 21.6 & 24.3 & 21.4 \\
Relative score & 18.9 & 20.9 & 21.3 & 22.6 & 25.9 & 21.9 \\
$\quad$ Minimum score & 18.8 & $\mathbf{2 1 . 2}$ & 22.2 & 22.2 & 24.5 & 21.8 \\
\hline Combining all except Historical Simulation & & & & & \\
$\quad$ Simple average & 18.7 & 20.4 & $\mathbf{2 2 . 4}$ & $\mathbf{2 3 . 3}$ & 26.2 & 22.2 \\
$\quad$ Relative score & 18.9 & $\mathbf{2 1 . 2}$ & 21.1 & 22.6 & 26.6 & 22.1 \\
$\quad$ Minimum score & $\mathbf{1 9 . 3}$ & 20.9 & 22.1 & 22.8 & $\mathbf{2 6 . 8}$ & $\mathbf{2 2 . 4}$ \\
\hline
\end{tabular}

Notes: The quantile score is presented in expression (1). Higher skill score values are better. Bold indicates best method in each column.

As we discussed in Sections 1 and 2, although ES is not elicitable, it is jointly elicitable with VaR. Therefore, in terms of scoring functions, we must evaluate ES jointly with VaR. We do this using the AL score. As the AL score takes negative values, we computed its skill score slightly differently to the quantile skill score. We calculated the $A L$ 
skill score for each method as the ratio of the score to that of the historical simulation method, then subtracted 1 from this ratio, and multiplied the result by 100 . Table 4 presents the AL skill scores for the $1 \%$ probability level.

\section{Table 4}

$1 \% \mathrm{VaR}$ and ES evaluated using AL skill score (\%).

\begin{tabular}{lcccccc}
\hline & CAC & DAX & FTSE & NIKKEI & S\&P & $\begin{array}{c}\text { Geo. } \\
\text { mean }\end{array}$ \\
\hline $\begin{array}{l}\text { Individual methods } \\
\text { Historical Simulation }\end{array}$ & 0.0 & 0.0 & 0.0 & 0.0 & 0.0 & 0.0 \\
GJR-GARCH & 6.6 & 6.4 & 8.6 & 12.9 & 8.9 & 8.7 \\
HAR-Range & 9.0 & $\mathbf{9 . 6}$ & 8.9 & 10.7 & $\mathbf{1 0 . 8}$ & 9.8 \\
CARE-AS & 7.6 & 7.6 & 8.9 & 13.2 & 10.6 & 9.5 \\
CAViaR-AS-EVT & 7.3 & 7.4 & 8.7 & 12.4 & 9.2 & 9.0 \\
\hline Combining all & & & & & & \\
$\quad$ Simple average & 8.7 & 9.0 & 9.2 & 13.0 & 10.2 & 10.0 \\
Relative score & 8.7 & 9.3 & 9.0 & 13.7 & 10.3 & 10.2 \\
$\quad$ Minimum score & 8.8 & 9.3 & $\mathbf{9 . 6}$ & 13.5 & 10.0 & 10.2 \\
\hline Combining all except Historical Simulation & & & & & \\
$\quad$ Simple average & 8.3 & 9.1 & 9.5 & $\mathbf{1 4 . 1}$ & 10.5 & 10.3 \\
Relative score & 8.7 & $\mathbf{9 . 6}$ & 8.9 & 13.7 & 10.6 & 10.3 \\
$\quad$ Minimum score & $\mathbf{9 . 1}$ & 9.3 & $\mathbf{9 . 6}$ & 13.8 & 10.6 & $\mathbf{1 0 . 5}$ \\
\hline
\end{tabular}

Notes: Table 1 defines the AL score, which is a version of the joint VaR and ES score of expression (2). Higher skill score values are better. Bold indicates best method in each column.

Table 5 summarises the out-of-sample results for the quantile score, and the four joint scoring functions that we presented in Table 1, which jointly evaluate VaR and ES forecast accuracy. For each scoring function, the table shows the skill scores averaged across the five indices. For the $1 \%$ probability level, the results for the quantile score and AL score were also reported in the final columns of Tables 3 and 4. As the NZ, FZG and AS scores are all positively valued, we computed their skill scores using the same approach that we had used for the quantile score. An alternative to our use of a finite set of joint scoring functions is proposed by Ziegel et al. (2017) who build on the work of Ehm et al. (2016) by using Murphy diagrams to establish whether one method dominates another in terms of a class of joint scoring functions. 
Table 5

VaR evaluated using quantile skill score (\%), and VaR and ES jointly evaluated using AL, NZ, FZG and AS skill scores (\%).

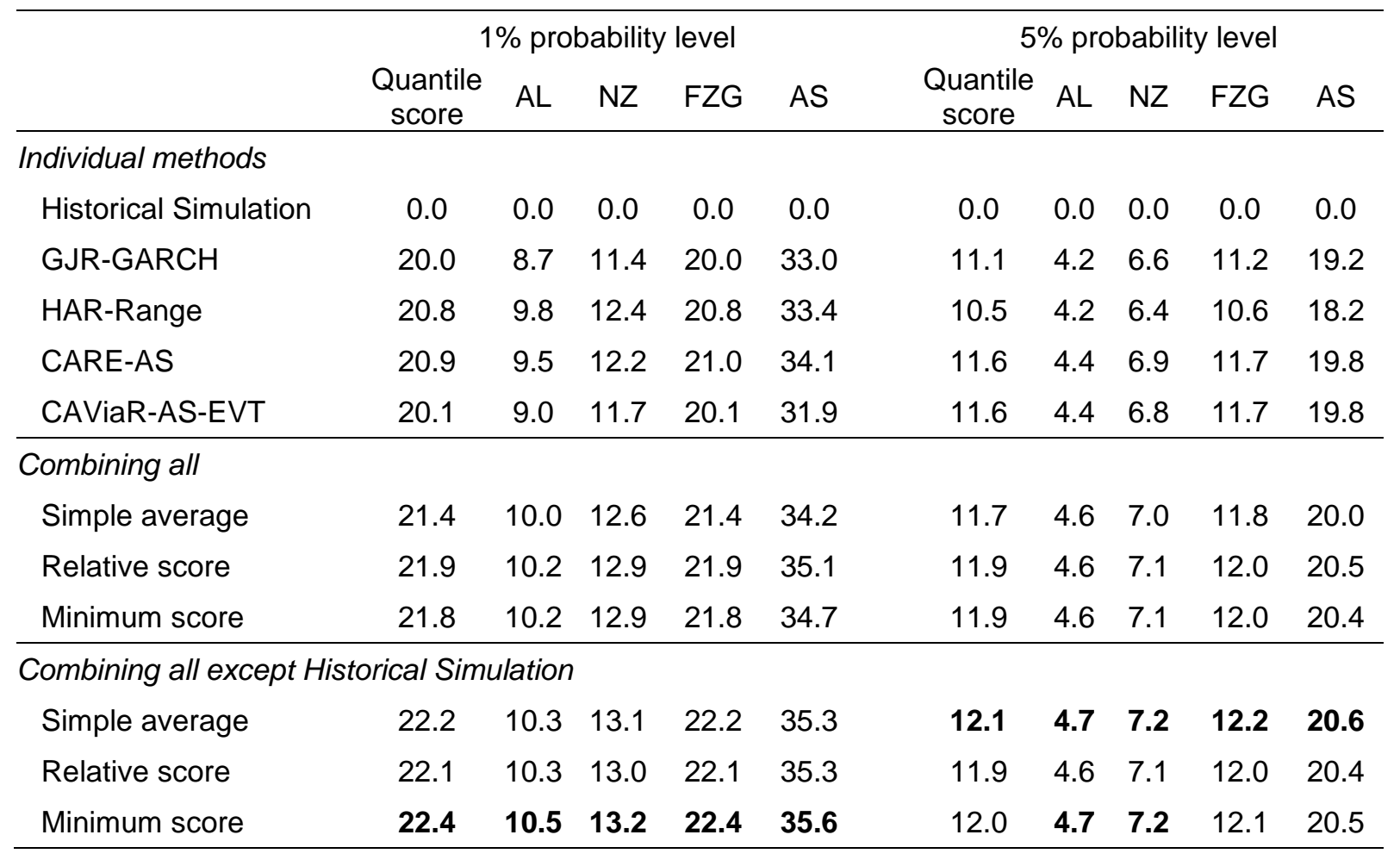

Notes: The quantile score is presented in expression (1). Table 1 defines the AL, NZ, FZG and AS scores, which are versions of the joint $\mathrm{VaR}$ and ES score of expression (2). The values presented here are the result of averaging skill scores across the five indices. Bold indicates best method in each column.

We have the following comments regarding Tables 3 to 5:

(i) The results are reasonably consistent across the five indices.

(ii) The results are reasonably consistent across the quantile score and the four joint VaR and ES scores.

(iii) For the $1 \%$ probability level, the best of the five individual methods were the HARRange approach and the CARE method.

(iv) For the 5\% probability level, the best of the five individual methods were the CARE and CAViaR-based methods.

(v) For both the $1 \%$ and 5\% probability levels, all of the combining methods outperformed all of the individual methods. 
(vi) For the $1 \%$ probability level, minimum score combining of the four competitive individual methods was the most accurate approach.

(vii) For the $5 \%$ probability level, the simple average of the four competitive individual methods was the most accurate approach, just slightly outperforming minimum score combining. To try to provide some intuition for why the simple average is more competitive for the $5 \%$ probability level than the $1 \%$ probability level, we first note that the other two combining methods were optimised by minimising the AL score. Looking at the columns of Table 5 corresponding to the AL score, we see that the results for the four sophisticated individual methods differ notably for the $1 \%$ probability level, but are quite similar for the $5 \%$ probability level. It is, therefore, not too surprising that the simple average was a reasonable approach for the $5 \%$ probability level, while a weighted average combination was preferable for the $1 \%$ probability level.

(viii) Perhaps unsurprisingly, the simple average was more affected by the inclusion of the historical simulation approach in the combination than the other combining methods. However, it is interesting to see that the other two combining methods were also affected to a degree, with the minimum score approach producing notably better results for the $1 \%$ probability level when historical simulation was excluded. This supports the view that it is unwise to include a poor method in a combination.

The model confidence set (MCS) testing framework of Hansen et al. (2011) enables a set of models to be obtained for which there is a pre-specified probability that the set contains the best model, when judged by a chosen loss function. If a model is not contained in the MCS, it is considered less likely to be the best model than those that are included in the MCS. We implemented MCS testing separately based on the quantile score and the four joint scoring functions, which jointly evaluate VaR and ES forecast accuracy. In each MCS test, we used the equivalence test based on the Diebold-Mariano test, and the one-sided elimination rule described as $T_{\max , M}$ by Hansen et al. (2011). We followed Hansen et al. 
(2011) by considering $75 \%$ and $90 \%$ confidence levels, and we report the results of the tests in Tables 6 and 7, respectively. For each scoring function, the tables report the number of indices for which each method was included in the MCS. As we have five indices in our study, the best possible value in each table is 5 . With five indices, five scoring functions, two probability levels and two confidence levels, we applied the MCS test 100 times. Historical simulation was clearly the worst method. The other four individual methods were included in most of the sets. This is perhaps not surprising, as we chose these methods because we felt they would be competitive, and hence may be useful in a combination. In the rows of Tables 6 and 7 corresponding to the combining methods, only one entry is not 5, indicating that the combining methods were excluded from just one MCS out of the 100 that we constructed.

\section{Table 6}

VaR evaluated using model confidence sets based on quantile score, and VaR and ES jointly evaluated using model confidence sets based on AL, NZ, FZG and AS scores. Values presented are the number of indices for which each method is within the model confidence set for $75 \%$ confidence level.

\begin{tabular}{|c|c|c|c|c|c|c|c|c|c|c|}
\hline & \multicolumn{5}{|c|}{$1 \%$ probability level } & \multicolumn{5}{|c|}{$5 \%$ probability level } \\
\hline & $\begin{array}{c}\text { Quantile } \\
\text { score }\end{array}$ & $\mathrm{AL}$ & $\mathrm{NZ}$ & $\mathrm{FZG}$ & AS & $\begin{array}{c}\text { Quantile } \\
\text { score }\end{array}$ & $\mathrm{AL}$ & $\mathrm{NZ}$ & $F Z G$ & AS \\
\hline \multicolumn{11}{|l|}{ Individual methods } \\
\hline Historical Simulation & 0 & 0 & 0 & 0 & 0 & 0 & 0 & 0 & 0 & 0 \\
\hline GJR-GARCH & 4 & 3 & 3 & 4 & 4 & 4 & 4 & 4 & 4 & 3 \\
\hline HAR-Range & 5 & 5 & 5 & 5 & 5 & 4 & 5 & 4 & 4 & 3 \\
\hline CARE-AS & 5 & 3 & 4 & 5 & 5 & 5 & 5 & 5 & 5 & 4 \\
\hline CAViaR-AS-EVT & 4 & 3 & 4 & 4 & 3 & 5 & 5 & 5 & 5 & 5 \\
\hline \multicolumn{11}{|l|}{ Combining all } \\
\hline Simple average & 5 & 5 & 5 & 5 & 5 & 5 & 5 & 5 & 5 & 5 \\
\hline Relative score & 5 & 5 & 5 & 5 & 5 & 5 & 5 & 5 & 5 & 5 \\
\hline Minimum score & 5 & 5 & 5 & 5 & 4 & 5 & 5 & 5 & 5 & 5 \\
\hline \multicolumn{11}{|c|}{ Combining all except Historical Simulation } \\
\hline Simple average & 5 & 5 & 5 & 5 & 5 & 5 & 5 & 5 & 5 & 5 \\
\hline Relative score & 5 & 5 & 5 & 5 & 5 & 5 & 5 & 5 & 5 & 5 \\
\hline Minimum score & 5 & 5 & 5 & 5 & 5 & 5 & 5 & 5 & 5 & 5 \\
\hline
\end{tabular}

Notes: The quantile score is presented in expression (1). Table 1 defines the AL, NZ, FZG and AS scores, which are versions of the joint VaR and ES score of expression (2). Higher values in this table are better, with 5 being the highest possible. 


\section{Table 7}

VaR evaluated using model confidence sets based on quantile score, and VaR and ES jointly evaluated using model confidence sets based on AL, NZ, FZG and AS scores. Values presented are the number of indices for which each method is within the model confidence set for $90 \%$ confidence level.

\begin{tabular}{|c|c|c|c|c|c|c|c|c|c|c|}
\hline & \multicolumn{5}{|c|}{$1 \%$ probability level } & \multicolumn{5}{|c|}{$5 \%$ probability level } \\
\hline & $\begin{array}{c}\text { Quantile } \\
\text { score }\end{array}$ & $\mathrm{AL}$ & $\mathrm{NZ}$ & $\mathrm{FZG}$ & AS & $\begin{array}{c}\text { Quantile } \\
\text { score }\end{array}$ & $\mathrm{AL}$ & $\mathrm{NZ}$ & $\mathrm{FZG}$ & AS \\
\hline \multicolumn{11}{|l|}{ Individual methods } \\
\hline Historical Simulation & 0 & 0 & 0 & 0 & 0 & 0 & 0 & 0 & 0 & 0 \\
\hline GJR-GARCH & 4 & 4 & 4 & 4 & 4 & 4 & 4 & 4 & 5 & 5 \\
\hline HAR-Range & 5 & 5 & 5 & 5 & 5 & 5 & 5 & 5 & 5 & 5 \\
\hline CARE-AS & 5 & 5 & 5 & 5 & 5 & 5 & 5 & 5 & 5 & 5 \\
\hline CAViaR-AS-EVT & 4 & 5 & 5 & 4 & 4 & 5 & 5 & 5 & 5 & 5 \\
\hline \multicolumn{11}{|l|}{ Combining all } \\
\hline Simple average & 5 & 5 & 5 & 5 & 5 & 5 & 5 & 5 & 5 & 5 \\
\hline Relative score & 5 & 5 & 5 & 5 & 5 & 5 & 5 & 5 & 5 & 5 \\
\hline Minimum score & 5 & 5 & 5 & 5 & 5 & 5 & 5 & 5 & 5 & 5 \\
\hline \multicolumn{11}{|c|}{ Combining all except Historical Simulation } \\
\hline Simple average & 5 & 5 & 5 & 5 & 5 & 5 & 5 & 5 & 5 & 5 \\
\hline Relative score & 5 & 5 & 5 & 5 & 5 & 5 & 5 & 5 & 5 & 5 \\
\hline Minimum score & 5 & 5 & 5 & 5 & 5 & 5 & 5 & 5 & 5 & 5 \\
\hline
\end{tabular}

Notes: The quantile score is presented in expression (1). Table 1 defines the AL, NZ, FZG and AS scores, which are versions of the joint VaR and ES score of expression (2). Higher values in this table are better, with 5 being the highest possible.

Our comparison of methods has been based on the final 2000 observations in each series. With the financial crisis starting not long before the start of this period, Fig. 1 shows that the first half of this period is perhaps more volatile than the second half. In Table 8 , we compare the results for the two halves of the out-of-sample period. The table focuses on the AL skill score, which evaluates both VaR and ES forecast accuracy. The results in this table confirm that the ranking of methods was broadly similar for each half of our out-of-sample period. 
Table 8

VaR and ES evaluated using AL skill score (\%) for different out-of-sample periods.

\begin{tabular}{|c|c|c|c|c|c|c|}
\hline & \multicolumn{3}{|c|}{$1 \%$ probability level } & \multicolumn{3}{|c|}{$5 \%$ probability level } \\
\hline & $\begin{array}{c}\text { Penultimate } \\
1000 \\
\text { days }\end{array}$ & $\begin{array}{l}\text { Final } \\
1000 \\
\text { days }\end{array}$ & $\begin{array}{c}\text { All } \\
2000 \\
\text { days }\end{array}$ & $\begin{array}{c}\text { Penultimate } \\
1000 \\
\text { days }\end{array}$ & $\begin{array}{l}\text { Final } \\
1000 \\
\text { days }\end{array}$ & $\begin{array}{l}\text { All } \\
2000 \\
\text { days }\end{array}$ \\
\hline \multicolumn{7}{|l|}{ Individual methods } \\
\hline Historical Simulation & 0.0 & 0.0 & 0.0 & 0.0 & 0.0 & 0.0 \\
\hline GJR-GARCH & 12.8 & 4.9 & 8.7 & 5.7 & 2.8 & 4.2 \\
\hline HAR-Range & 13.6 & 6.4 & 9.8 & 5.8 & 2.7 & 4.2 \\
\hline CARE-AS & 14.3 & 5.2 & 9.5 & 6.5 & 2.6 & 4.4 \\
\hline CAViaR-AS-EVT & 12.8 & 5.6 & 9.0 & 6.1 & 2.8 & 4.4 \\
\hline \multicolumn{7}{|l|}{ Combining all } \\
\hline Simple average & 13.9 & 6.6 & 10.0 & 6.4 & 2.9 & 4.6 \\
\hline Relative score & 14.2 & 6.5 & 10.2 & 6.4 & 3.0 & 4.6 \\
\hline Minimum score & 14.2 & 6.7 & 10.2 & 6.5 & 3.0 & 4.6 \\
\hline \multicolumn{7}{|c|}{ Combining all except Historical Simulation } \\
\hline Simple average & 14.5 & 6.5 & 10.3 & 6.5 & 3.1 & 4.7 \\
\hline Relative score & 14.4 & 6.6 & 10.3 & 6.3 & 3.0 & 4.6 \\
\hline Minimum score & 14.7 & 6.7 & 10.5 & 6.5 & 3.0 & 4.7 \\
\hline
\end{tabular}

Notes: Table 1 defines the AL score, which is a version of the joint VaR and ES score of expression (2). The values presented here are the result of averaging skill scores across the five indices. Higher skill score values are better. Bold indicates best method in each column.

\section{Simulation Study}

To investigate further the combining methods, we implemented an empirical study using data simulated from the following three data generating processes (DGP):

DGP1: This is a GJR-GARCH $(1,1)$ process with Student $t$ distribution. We chose the parameters to be the average of the 2000 sets of parameter values that we had estimated using the 2000 rolling windows for the filtered FTSE 100 returns.

DGP2: This process and DGP3 were used by Manganelli and Engle (2001) in their study of VaR and ES. For DGP2, we generated data from a GJR-GARCH(1,1) process with error term alternatively drawn from a Student $t$ distribution with 3 degrees of freedom and a Gamma distribution with shape and scale parameters equal to 2 and 0.5 , respectively. The 
values drawn from the Gamma distribution were standardised so that they had zero mean and unit variance. We used the same GJR-GARCH model parameters as in DGP1.

DGP3: This process was based on an asymmetric slope CAViaR process with a probability level of $15 \%$. We chose this model because it is used in one of the individual methods included in our empirical analysis. We used parameters that were the average of those estimated using the rolling windows for the filtered FTSE 100 returns. Starting with initial values $q_{0}$ and $y_{0}$ for the quantile and the observation, we used the CAViaR process to generate $q_{1}$, the value of the quantile for the first period. The simulated value $y_{1}$ for this period was then generated from a distribution with quantile equal to $q_{1}$. This was achieved by multiplying a randomly generated value by a standard deviation equal to $q_{1}$ divided by the quantile of the distribution used to generate the random value. To generate the random values, we sequentially used Student $t$ distributions with 3 and 4 degrees of freedom, and a Gamma distribution with shape and scale parameters equal to 2 and 0.5 , respectively. We repeated this procedure using $q_{t-1}$ and $y_{t-1}$ in the CAViaR model to generate $q_{t}$, which is then used with a randomly sampled value to produce $y_{t}$.

We generated one series of 6000 observations from each of the three DGPs. The structure of our study matched our analysis of the stock indices, with rolling windows of 2000 periods used for repeated re-estimation of parameters. We used the final 2000 periods to compare out-of-sample forecast accuracy. We implemented the same individual methods that we considered for the stock indices, with the omission of the HAR-Range method, which relies on intraday data. We considered combinations of all four individual methods, as well as combinations that excluded historical simulation. Tables 9 to 11 present out-of-sample skill scores for the simulated series. We have the following comments regarding these results:

(i) For DGP1, the GJR-GARCH model is optimal, and so it was to be expected that this method performed well. But it is noticeable that the results were matched by those from the relative score and minimum score combining methods. 
(ii) For DGP1, the simple average benefitted from the removal of historical simulation. For DGP2 and DGP3, this was the case for only the $5 \%$ probability level.

(iii) For all three processes, removing historical simulation from the combination did not noticeably improve the results of the relative score and minimum score combining methods.

(iv) For DGP2, simple average combining was the best method for the $1 \%$ probability level. For the 5\% probability level, all combining methods performed well, with the best results achieved by the simple average with historical simulation excluded.

(v) Given the nature of DGP3, it is not surprising to see that the CAViaR-AS-EVT method performed well for this process. However, comparable results were achieved by simple average combining for the $1 \%$ probability level. For the $5 \%$ probability level, all the combining methods performed very well, with the exception of the simple average with historical simulation included.

\section{Table 9}

For simulated data from DGP1, VaR evaluated using quantile skill score (\%), and VaR and ES jointly evaluated using AL, NZ, FZG and AS skill scores (\%).

\begin{tabular}{|c|c|c|c|c|c|c|c|c|c|c|}
\hline & \multicolumn{5}{|c|}{$1 \%$ probability level } & \multicolumn{5}{|c|}{$5 \%$ probability level } \\
\hline & $\begin{array}{c}\text { Quantile } \\
\text { score }\end{array}$ & $\mathrm{AL}$ & $\mathrm{NZ}$ & $F Z G$ & AS & $\begin{array}{c}\text { Quantile } \\
\text { score }\end{array}$ & $\mathrm{AL}$ & $\mathrm{NZ}$ & $F Z G$ & AS \\
\hline \multicolumn{11}{|l|}{ Individual methods } \\
\hline Historical Simulation & 0.0 & 0.0 & 0.0 & 0.0 & 0.0 & 0.0 & 0.0 & 0.0 & 0.0 & 0.0 \\
\hline GJR-GARCH & 25.1 & 8.9 & 14.1 & 24.9 & 41.1 & 14.2 & 4.7 & 8.1 & 14.1 & 23.0 \\
\hline CARE-AS & 24.2 & 8.6 & 13.5 & 24.0 & 40.5 & 13.8 & 4.5 & 7.9 & 13.7 & 22.2 \\
\hline CAViaR-AS-EVT & 23.3 & 8.3 & 13.1 & 23.1 & 37.7 & 14.0 & 4.6 & 8.0 & 13.9 & 22.5 \\
\hline \multicolumn{11}{|l|}{ Combining all } \\
\hline Simple average & 21.9 & 7.7 & 12.3 & 21.7 & 36.4 & 12.9 & 4.1 & 7.2 & 12.8 & 21.5 \\
\hline Relative score & 25.2 & 8.9 & 14.1 & 25.0 & 41.1 & 14.2 & 4.7 & 8.1 & 14.1 & 23.0 \\
\hline Minimum score & 24.4 & 8.7 & 13.7 & 24.2 & 39.5 & 14.1 & 4.6 & 8.1 & 14.0 & 22.9 \\
\hline \multicolumn{11}{|c|}{ Combining all except Historical Simulation } \\
\hline Simple average & 25.1 & 8.8 & 14.0 & 24.8 & 41.8 & 14.1 & 4.6 & 8.1 & 14.0 & 22.7 \\
\hline Relative score & 25.2 & 8.9 & 14.1 & 25.0 & 41.1 & 14.2 & 4.7 & 8.1 & 14.1 & 23.0 \\
\hline Minimum score & 25.2 & 8.9 & 14.1 & 24.9 & 41.2 & 14.2 & 4.7 & 8.1 & 14.1 & 23.0 \\
\hline
\end{tabular}

Notes: The quantile score is presented in expression (1). Table 1 defines the AL, NZ, FZG and AS scores, which are versions of the joint VaR and ES score of expression (2). Higher skill score values are better. Bold indicates best method in each column. 


\section{Table 10}

For simulated data from DGP2, VaR evaluated using quantile skill score (\%), and VaR and ES jointly evaluated using AL, NZ, FZG and AS skill scores (\%).

\begin{tabular}{|c|c|c|c|c|c|c|c|c|c|c|}
\hline & \multicolumn{5}{|c|}{$1 \%$ probability level } & \multicolumn{5}{|c|}{$5 \%$ probability level } \\
\hline & $\begin{array}{c}\text { Quantile } \\
\text { score }\end{array}$ & $\mathrm{AL}$ & $\mathrm{NZ}$ & $F Z G$ & AS & $\begin{array}{c}\text { Quantile } \\
\text { score }\end{array}$ & $\mathrm{AL}$ & $\mathrm{NZ}$ & $F Z G$ & AS \\
\hline \multicolumn{11}{|l|}{ Individual methods } \\
\hline Historical Simulation & 0.0 & 0.0 & 0.0 & 0.0 & 0.0 & 0.0 & 0.0 & 0.0 & 0.0 & 0.0 \\
\hline GJR-GARCH & 14.4 & 8.7 & 10.0 & 14.5 & 19.8 & 10.5 & 5.4 & 7.4 & 10.6 & 12.6 \\
\hline CARE-AS & 14.8 & 8.0 & 9.7 & 14.9 & 18.2 & 10.6 & 5.3 & 7.3 & 10.8 & 12.9 \\
\hline CAViaR-AS-EVT & 14.8 & 8.9 & 10.4 & 15.0 & 17.5 & 10.2 & 5.3 & 7.2 & 10.3 & 11.6 \\
\hline \multicolumn{11}{|l|}{ Combining all } \\
\hline Simple average & 16.8 & 9.4 & 11.2 & 16.9 & 22.2 & 9.8 & 5.1 & 7.0 & 9.9 & 12.1 \\
\hline Relative score & 14.7 & 8.8 & 10.2 & 14.8 & 20.1 & 10.7 & 5.5 & 7.6 & 10.8 & 12.9 \\
\hline Minimum score & 14.9 & 8.7 & 10.2 & 15.0 & 20.1 & 10.5 & 5.4 & 7.4 & 10.7 & 12.8 \\
\hline \multicolumn{11}{|c|}{ Combining all except Historical Simulation } \\
\hline Simple average & 16.2 & 9.5 & 11.1 & 16.4 & 20.4 & 10.8 & 5.6 & 7.7 & 11.0 & 13.0 \\
\hline Relative score & 14.6 & 8.7 & 10.2 & 14.8 & 20.1 & 10.7 & 5.5 & 7.6 & 10.8 & 12.9 \\
\hline Minimum score & 14.6 & 8.6 & 10.0 & 14.7 & 19.4 & 10.7 & 5.5 & 7.5 & 10.8 & 12.9 \\
\hline
\end{tabular}

Notes: The quantile score is presented in expression (1). Table 1 defines the AL, NZ, FZG and AS scores, which are versions of the joint VaR and ES score of expression (2). Higher skill score values are better. Bold indicates best method in each column.

\section{Table 11}

For simulated data from DGP3, VaR evaluated using quantile skill score (\%), and VaR and ES jointly evaluated using AL, NZ, FZG and AS skill scores (\%).

\begin{tabular}{|c|c|c|c|c|c|c|c|c|c|c|}
\hline & \multicolumn{5}{|c|}{$1 \%$ probability level } & \multicolumn{5}{|c|}{$5 \%$ probability level } \\
\hline & $\begin{array}{c}\text { Quantile } \\
\text { score }\end{array}$ & AL & $\mathrm{NZ}$ & $F Z G$ & AS & $\begin{array}{c}\text { Quantile } \\
\text { score }\end{array}$ & $\mathrm{AL}$ & $\mathrm{NZ}$ & $\mathrm{FZG}$ & AS \\
\hline \multicolumn{11}{|l|}{ Individual methods } \\
\hline Historical Simulation & 0.0 & 0.0 & 0.0 & 0.0 & 0.0 & 0.0 & 0.0 & 0.0 & 0.0 & 0.0 \\
\hline GJR-GARCH & 17.6 & 13.1 & 12.7 & 17.8 & 23.1 & 13.4 & 6.8 & 9.0 & 13.5 & 16.8 \\
\hline CARE-AS & 18.6 & 13.8 & 13.4 & 18.7 & 22.9 & 12.8 & 6.5 & 8.6 & 12.9 & 16.1 \\
\hline CAViaR-AS-EVT & 19.4 & 14.8 & 14.2 & 19.6 & 23.8 & 13.5 & 7.2 & 9.3 & 13.6 & 16.5 \\
\hline \multicolumn{11}{|l|}{ Combining all } \\
\hline Simple average & 19.4 & 13.6 & 13.5 & 19.5 & 24.9 & 12.8 & 6.3 & 8.5 & 12.9 & 17.0 \\
\hline Relative score & 18.3 & 13.5 & 13.2 & 18.5 & 22.5 & 13.4 & 6.9 & 9.1 & 13.6 & 17.0 \\
\hline Minimum score & 18.6 & 13.5 & 13.2 & 18.7 & 23.5 & 13.5 & 6.9 & 9.1 & 13.6 & 17.1 \\
\hline \multicolumn{11}{|c|}{ Combining all except Historical Simulation } \\
\hline Simple average & 19.7 & 14.6 & 14.2 & 19.8 & 24.7 & 13.7 & 7.1 & 9.3 & 13.8 & 17.2 \\
\hline Relative score & 18.5 & 13.9 & 13.5 & 18.7 & 23.1 & 13.5 & 7.1 & 9.2 & 13.6 & 16.9 \\
\hline Minimum score & 17.8 & 13.5 & 13.0 & 18.0 & 21.6 & 13.6 & 7.1 & 9.3 & 13.7 & 17.0 \\
\hline
\end{tabular}




\section{Summary and Concluding Comments}

In this paper, we have introduced forecast combination to ES prediction. As ES is not elicitable, we simultaneously estimate combining weights for VaR and ES using recently proposed joint scoring functions. Our minimum score combining approach allows convex combining weights to differ for $\mathrm{VaR}$ and ES prediction. We also considered a relative score combining approach that has the appeal of requiring the estimation of just one parameter.

In our empirical study of stock indices, to compare the methods, we focused on the scoring functions. We combined parametric, nonparametric and semiparametric time series methods, as well as a method based on the intraday range. Looking at the average performance across our set of stock indices, we found that all of the individual methods were outperformed by all of the combining methods. For the $1 \%$ probability level, the best results were provided by weighted combining approaches. The simple average also performed well, particularly for the 5\% probability level, provided the poorly performing historical simulation method was omitted from the combination. A simulation study provided support for the combining methods.

In future work, it would be interesting to consider alternative combining methods, and different sets of individual methods. Multi-step ahead prediction is another potential area for future work. Combining weights for multiple lead times could be estimated by minimising a joint score summed over different lead times, or the weights could be estimated separately for each lead time, which seems appealing as the relative performance of methods can vary across lead times. It would also be interesting to compare forecast accuracy using the Murphy diagrams of Ziegel et al. (2017), and perhaps also measures of economic significance.

\section{Acknowledgements}

We are very grateful to an associate editor and two referees for providing comments that helped greatly to improve the paper. 


\section{References}

Acerbi, C., \& Székeley, B. (2014). Backtesting expected shortfall, Risk, December, 76-81.

Alizadeh, S., Brandt, M.W., \& Diebold, F.X. (2002). Range-based estimation of stochastic volatility models. Journal of Finance, 57(3), 1047-1091.

Artzner, P., Delbaen, F., Eber, J.M., \& Heath, D. (1999). Coherent measures of risk. Mathematical Finance, 9(3), 203-228.

Basel Committee (2016). Minimum capital requirements for Market Risk. Technical report, Basel Committee on Banking Supervision.

Bates, J.M., \& Granger, C.W.J. (1969). The combination of forecasts. Operational Research Quarterly, 20(4), 451-468.

Brownlees, C. T., \& Gallo, G.M. (2010). Comparison of volatility measures: A risk Management perspective. Journal of Financial Econometrics, 8(1), 29-56.

Chen, C.W.S., Gerlach, R., Hwang, B.B.K., McAleer, M. (2012). Forecasting Value-at-Risk using nonlinear regression quantiles and the intra-day range. International Journal of Forecasting, 28(3), 557-574.

Corsi, F. (2009). A simple approximate long-memory model of realized volatility. Journal of Financial Econometrics, 7(2), 174-196.

Dimitriadis, T., \& Bayer, S. (2017). A joint quantile and expected shortfall regression framework. Working paper, arXiv:1704.02213v3.

Efron, B. (1991). Regression percentiles using asymmetric squared error loss. Statistica Sinica, $1,93-125$

Ehm, W., Gneiting, T., Jordan, A., \& Krüger, F. (2016). Of quantiles and expectiles: Consistent scoring functions, Choquet representations and forecast rankings. Journal of the Royal Statistical Society: Series B (Statistical Methodology), 78(3), 505-562.

Elliott, G., \& Timmermann, A. (2004). Optimal forecast combinations under general loss functions and forecast error distributions. Journal of Econometrics, 122(1), 47-80. 
Engle, R.F., \& Manganelli, S. (2004). CAViaR: Conditional autoregressive value at risk by regression quantiles. Journal of Business and Economic Statistics, 22(4), 367-381.

Escanciano, J. C., \& Olmo, J. (2010). Backtesting parametric value-at-risk with estimation risk. Journal of Business and Economic Statistics, 28(1), 36-51.

Fissler, T., \& Ziegel, J.A. (2016). Higher order elicitability and Osband's Principle. Annals of Statistics, 44(4), 1680-1707.

Fissler, T., Ziegel, J.A., \& Gneiting, T. (2016). Expected shortfall is jointly elicitable with value at risk - implications for backtesting. Risk, January, 58-61.

Fuertes, A.-M., \& Olmo, J. (2013). Optimally harnessing inter-day and intra-day information for daily value-at-risk prediction. International Journal of Forecasting, 29(1), 28-42.

Gerlach, R., \& Chen, C.W. (2014). Bayesian expected shortfall forecasting incorporating the intraday range. Journal of Financial Econometrics, 14(1), 128-158.

Giacomini, R., \& Komunjer, I. (2005). Evaluation and combination of conditional quantile forecasts. Journal of Business and Economic Statistics, 23(4), 416-431.

Gneiting, T. (2011). Making and evaluating point forecasts. Journal of the American Statistical Association, 106(494), 746-762.

Gneiting, T., \& Raftery, A.E. (2007). Strictly proper scoring rules, prediction, and estimation. Journal of the American Statistical Association, 102(477), 359-378.

Granger, C.W. J. (1989). Combining forecasts - twenty years later. Journal of Forecasting, $8(3), 167-173$.

Granger, C.W.J., White, H., \& Kamstra, M.J. (1989). Interval forecasting: An analysis based upon ARCH-quantile estimators. Journal of Econometrics, 40(1), 87-96.

Halbleib, R., \& Pohlmeier, W. (2012). Improving the value at risk forecasts: Theory and evidence from financial crisis. Journal of Economic Dynamics and Control, 36(8), 12121228. 
Hall, S. G., \& Mitchell, J. (2007). Combining density forecasts. Journal of Forecasting, 23(1), 1-13.

Hansen, P.R., Lunde, A., \& Nason, J.M. (2011). The model confidence set. Econometrica, $79(2), 453-497$.

Jalal, A., \& Rockinger, M. (2008). Predicting tail-related risk measures: The consequences of using GARCH filters for non-GARCH data. Journal of Empirical Finance, 15(5), 868877.

Jeon, J., \& Taylor, J.W. (2013). Using implied volatility with CAViaR models for value at risk estimation. Journal of Forecasting, 32(1), 62-74.

Jore, A. S., Mitchell, J., \& Vahey, S. P. (2010). Combining forecast densities from VARs with uncertain instabilities. Journal of Applied Econometrics, 25(4), 621-634.

Manganelli, S., \& Engle, R.F. (2004). A comparison of value-at-risk models in finance. In G. Szegö (ed.), Risk Measures for the 21 st Century, pp. 123-144, Chichester, Wiley.

McAleer, M., Jiménez-Martín, J.-A., \& Pérez-Amaral, T. (2013a). Has the Basel Accord improved risk management during the Global Financial Crisis? The North American Journal of Economics and Finance, 26(C), 250-265.

McAleer, M., Jiménez-Martín, J.-A., \& Pérez-Amaral, T. (2013b). International evidence on GFC-robust forecasts for risk management under the Basel Accords. Journal of Forecasting, 32(3), 267-288.

McNeil, A.J., \& Frey, R. (2000). Estimation of tail-related risk measures for heteroscedastic financial time series: An extreme value approach. Journal of Empirical Finance, 7(3-4), 271-300.

Opschoor, A., Van Dijk, D., \& van der Wel, M. (2017). Combining density forecasts using focused scoring rules. Journal of Applied Econometrics, 32(7), 1298-1313.

Newey, W.K., \& Powell, J.L. (1987). Asymmetric least squares estimation and testing. Econometrica, 55(4), 819-847. 
Nieto, M.R., \& Ruiz, E. (2016). Frontiers in VaR forecasting and backtesting. International Journal of Forecasting, 32(2), 475-501.

Nolde, N., \& Ziegel, J.F. (2017). Elicitiability and backtesting: Perspectives for banking regulation. Annals of Applied Statistics, 11(4) 1833-1874.

Patton, A.J., Ziegel, J.F., \& Chen, R. (2019). Dynamic semiparametric models for expected shortfall (and value-at-risk). Journal of Econometrics, forthcoming.

Shan, K., \& Yang, Y. (2009). Combining regression quantile estimators. Statistica Sinica, $1171-1191$.

Stock, J.H., \& Watson, M. (2001). A comparison of linear and nonlinear univariate models for forecasting macroeconomic time series. In R.F. Engle, \& White, H. (eds.), Festschrift in Honour of Clive Granger, pp. 1-44. Cambridge, Cambridge University Press.

Taylor, J.W. (2008). Estimating value at risk and expected shortfall using expectiles. Journal of Financial Econometrics, 6(2), 231-252.

Taylor, J.W. (2019). Forecasting value at risk and expected shortfall using a semiparametric approach based on the asymmetric Laplace distribution. Journal of Business and Economic, Statistics, 37(1) 121-133.

Taylor, J.W., \& Bunn, D.W. (1998). Combining forecast quantiles using quantile regression: Investigating the derived weights, estimator bias and imposing constraints. Journal of Applied Statistics, 25(2), 193-206.

Ziegel, J.F., Krüger, F., Jordan, A., \& Fernando, F. (2017). Murphy diagrams: Forecast evaluation of expected shortfall. Working paper, arXiv:1705.04537. 